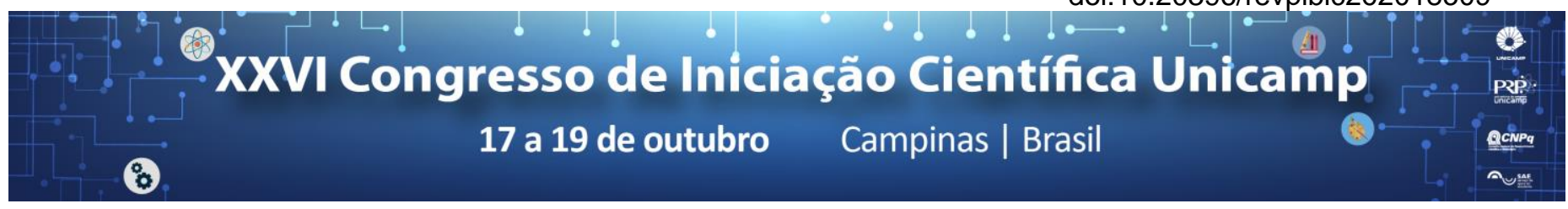

\title{
Evaluation of the effect of culture supernatant of keratinocytes in hyperalgesia model
}

\author{
Cíntia A. Souza*, Carlos A. Parada*, Gilson G. dos Santos, Felipe H. Farias
}

\begin{abstract}
Inflammation is a defense response against human death by physical, chemical or biological agents, which triggers, among other factors, pain. Despite being a warning and protection signal, it decreases the life quality.In view of this, research into new antinociceptive therapeutic options is necessary. As it was verified that the keratinocytes of the basal stratum of the epidermis release endogenous opioid, being an important antinociceptive mechanism, the best understanding of the relationship between peripheral nervous system, skin (keratinocytes) and analgesia is important.
\end{abstract}

\section{Key words:}

inflammatory hyperalgesia, opioid, keratinocytes.

\section{Introduction}

Inflammation is a defense response against human death by physical, chemical or biological agents, which triggers, among other factors, pain, as a warning and protection signal. Despite being an important alertness and protection mechanism, pain provides suffering, which decreases the quality of life ${ }^{1}$. Therefore, it is known that endogenous mechanisms that counteract pain and inflammation, such as the release of endogenous opioids by immune cells, are produced ${ }^{2}$. Among such opioids, bendorphin is notable for its antinociceptive potential. As it was verified that the keratinocytes of the basal stratum of the epidermis are also responsible for the release of such opioid, this direct relationship between the peripheral nervous system and keratinocytes is evidenced as a promising research field for different forms of analgesia, mainly because of its local action ${ }^{3}$. Thus, the present study aims to evaluate the analgesic potential of culture supernatant of human keratinocytes in the experimental model of inflammatory hyperalgesia. The purpose of it is to establish the anti-hyperalgesic mechanism and time of action, which may bring a new way of local treatment of inflammatory hyperalgesia.

\section{Results and Discussion}

Male albino Wistar rats (150-200 g) obtained from CEMIB/UNICAMP were used. Prior to the study, an electronic test of mechanical hyperalgesia (von Frey) was performed. After 1 hour, carrageenan (100 ug/50 uL/paw) was administered subcutaneously in the intraplate region of the right hind paw. After 2 hours of administration of the hyperalgesic agent, $50 \mu \mathrm{L}$ of keratinocyte culture supernatant (treated groups, $n=6$ ) of 1 or 3 days of culture or $50 \mu \mathrm{L}$ of keratinocyte culture medium (control group, $n=6$ ) was administered in the periods described above. After $0.5,1,2,4$ and 6 hours of supernatant administration, the mechanical hyperalgesia was assessed by the von Frey test. Administration of carrageenan (100 ug/50 uL/paw) in the subcutaneous tissue induced hyperalgesia for 6 hours evaluated. In order to obtain the antihyperalgesic effect of keratinocyte culture supernatant, the supernatant was administered (SCQ / $50 \mu \mathrm{L})$. The pre-treatment SCQ $(50 \mu \mathrm{L})$ of days 1 and 3 reversed carrageenan-induced hyperalgesia 30 minutes after administration, and from 30 minutes to one hour after administration a reduction of hyperalgesia could be verified until it was reestablished (1 hour after). Local administration of carrageenan (100 $\mu \mathrm{g} / \mathrm{paw})$ in the subcutaneous tissue of rat hind paw induced mechanical hyperalgesia evaluated $3 \mathrm{~h}$ after its injection. After 2 hours of administration of the hyperalgesic agent, a nonselective opioid antagonist (Naloxone) was administered at the same site. After 2.5 hours, $50 \mu \mathrm{L}$ of keratinocyte culture supernatant (treated groups, $n=6$ ) of 3 days or 50 $\mu \mathrm{L}$ of keratinocyte culture medium (control group, $n=6$ ) was administered at the same site. As shown in figure 1, the administration of naloxone inihibited the antihyperalgesic effect of the keratinocyte culture supernatant, confirming the release of endogenous opioid by keratinocytes.

Image 1. The local administration of keratinocyte cell culture supernatant in peripheral tissue reduces the carrageenan-induced hyperalgesia, which was inihibited by the administration of naloxone (Nalo).

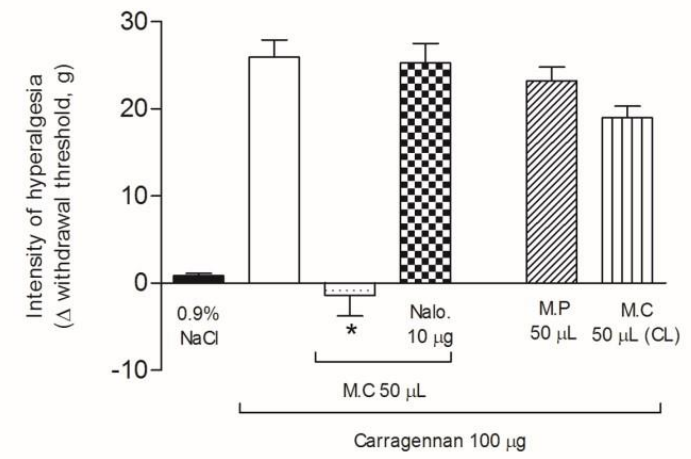

\section{Conclusions}

The keratinocytes culture supernatant of 1 and 3 day reversed, completely, the inflammatory hyperalgesia induced by carrageenan, from 30 minutes to 1 hour after its application. The reversal of inflammatory hyperalgesia is mediated by endogenous opioids released by keratinocytes. In addition, there is no difference in time and duration of reversal of hyperalgesia between cultures of 1 or 3 days.

\section{Acknowledgement Supported By PIBIC/SAE-Brazil (UNICAMP).}

${ }^{1}$ Stüve O, Warnke C, Deason K, Stangel M, Kieseier BC, Hartung H-P, et al CD19 as a molecular target in CNS autoimmunity. Acta Neuropathol 2014; 128: 177-90.

${ }^{2}$ Woolf, C.J. - Recent advances in the pathophysiology of acute pain. Br. J. Anaesth., 1989; 63:139146.

${ }^{3}$ Paul L.; Mei B.; Stanislaus B.; Theo R. Expression of $\mu$-Opiate Receptor in Human Epidermis and Keratinocytes. Journal of Investigative Dermatology. Volume 111, Issue 2, August 1998, Pages 297-301. 